EOMmUn Communication et organisation

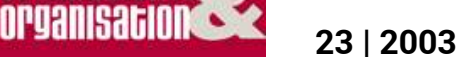

Modèles de communication Homme-Animal

\title{
Rencontre avec Hubert Montagner
}

Hélène Dufau

\section{OpenEdition}

Journals

Édition électronique

URL : http://journals.openedition.org/communicationorganisation/2865

DOI : 10.4000/communicationorganisation.2865

ISSN : 1775-3546

Éditeur

Presses universitaires de Bordeaux

Édition imprimée

Date de publication : 1 mai 2003

ISSN : 1168-5549

\section{Référence électronique}

Hélène Dufau, « Rencontre avec Hubert Montagner », Communication et organisation [En ligne],

23 | 2003, mis en ligne le 27 mars 2012, consulté le 20 avril 2019. URL : http://

journals.openedition.org/communicationorganisation/2865; DOI : 10.4000/

communicationorganisation.2865

Ce document a été généré automatiquement le 20 avril 2019

(c) Presses universitaires de Bordeaux 


\title{
Rencontre avec Hubert Montagner
}

\author{
Hélène Dufau
}

\section{NOTE DE L'ÉDITEUR}

Propos recueillis par Hélène Dufau le 01/04/2003

1 Hubert Montagner est directeur de recherche à l'Institut National de la Santé et de la Recherche Médicale (INSERM) où il a la responsabilité du groupe de recherche « Psychophysiologie et psychopathologie du développement » (UMR-CNRS 554 Université Victor Ségalen-Bordeaux 2). Expert à l'Institut National de la Recherche Pédagogique auprès de la «Mission École Primaire » du Comité National de Suivi de la Charte «Bâtir l'école du XXI ${ }^{e}$ siècle», il est aussi appelé fréquemment en tant qu'expert et/ou consultant auprès d'institutions et de gouvernements étrangers. Ainsi collabore-t-il actuellement avec le gouvernement du Brésil et le Medical Science Monitor de New-York. Outre l'Université Bordeaux 2, l'université Pierre et Marie Curie (Paris. Faculté de médecine La Pitié Salpêtrière) et l'Université François Rabelais de Tours bénéficient de ses enseignements. Il est également régulièrement professeur invité à l'Institut Supérieur des Sciences de l'Information de l'Université de Coimbra (Portugal) et aux facultés de médecine des universités de Brasilia, Porto Alegre et Rio de Janeiro (Brésil).

Hélène Dufau. : L'idée qui a sous-tendu la préparation de ce numéro est que dans la communication humaine, on retrouve un certain nombre de facultés, de comportements, que l'homme a en partage avec l'animal. Se tourner vers les dimensions animales de la communication, qui se manifestent notamment dans la communication homme/animal peut-il éclairer certaines réalités de la communication humaine, et parfois même contribuer à l'amender ?

Hubert Montagner: Oui, je viens de publier chez Odile Jacob un ouvrage. L'enfant et l'animal (sept. 2002). Alors je définis tout d'abord, dans une première approche, les distinctions que je fais entre l'animal utilitaire, l'animal familier, l'animal de compagnie. L'animal sauvage est exclu de cette problématique. L'animal utilitaire se situe dans un rapport où l'homme et l'animal sont économiquement interdépendants. Il peut 
néanmoins devenir un animal de compagnie ou un animal familier. L'animal de compagnie, lui, c'est un compagnon, celui " avec qui on mange son pain", avec qui on partage le gite et le couvert. $11 \mathrm{y}$ en a certains que l'homme choisit pour vivre dans sa mouvance immédiate, pour sa sécurité physique et affective, pour se protéger contre le monde extérieur ou contre ce qu'il perçoit d'insécurisant dans le monde extérieur. C'est là une première motivation.

La deuxième motivation, c'est la recherche de la valorisation personnelle : c'est l'animal " potiche/miroir », que l'homme exhibe, met en scène, pour se valoriser lui-même (aspect narcissique) ; et il finit par y avoir une ressemblance entre l'animal et l'humain, au plan des comportement, de la façon de s'habiller. C'est le cas bien connu du propriétaire qui met un ruban sur la tête du chien et du chat, de la même couleur, de la même texture que le ruban qu'elle met elle-même dans ses cheveux... c'est un cas bien connu.

Il y a donc deux types d'animaux parmi les animaux de compagnie.

6 L'animal familier, lui, a une familiarité de tous les instants ; c'est celui qui fait partie de la famille. Par opposition avec l'animal de compagnie, on le laisse s'exprimer librement, au moins à certains moments ; tandis que l'animal de compagnie, on veut l'avoir à sa botte. Il faut le conditionner et on le tyrannise, à la limite, s'il ne fait pas ce qu'on veut qu'il fasse. L'animal familier, c'est un partenaire qui par sa façon d'être, son comportement, donne l'impression que c'est un partenaire quasiment humain. C'est avec l'animal familier qu'il est le plus probable que l'on ait une relation de confiance, de confident, de complicité. C'est avec lui que l'enfant peut avoir une relation de partenariat qui va lui permettre de rétablir des fonctionnements cérébraux harmonieux.

7 Le comportement du cerveau peut être représenté par la métaphore de la fusée : au premier niveau, il y a la mise à feu. En fait, ce que l'animal apporte à l'enfant se situe dans la métaphore de la fusée : rien n'est possible sans la mise à feu. L'enfant doit s'installer sur le versant de la sécurité affective. Un enfant insécurisé ne peut pas révéler ses potentialités et ses compétences. L'animal présente un avantage, c'est un partenaire qui ne parle pas, ne juge pas, ne trahit pas, ne renvoie pas aux difficultés. Au contraire, il a l'air d'écouter, d'entendre, d'être toujours d'accord. En fait, la relation avec l'animal est anxiolytique. Quand un enfant caresse un chien, on observe une diminution significative de son rythme cardiaque (c'est la même chose pour un adulte).

8 Deuxième niveau de la fusée, c'est quand la mise à feu est réalisée, que l'enfant a pu s'installer sur le versant de la sécurité affective, et qu'il a déverrouillé son intérieur. Alors, il peut libérer son extérieur. Cette dimension comprend deux facettes interactives. Il peut sans retenue libérer ses émotions; c'est ce qu'il fait en le disant à l'animal : «tu sais, je suis content parce que..., je suis un peu triste parce que maman, parce que papa... j'ai eu peur..."

9 Dans ce cas, il va pouvoir libérer les 6 émotions considérées comme fondamentales, universelles et innées à l'espèce humaine : la joie, la colère, la peur, la tristesse, le dégoût et la surprise.

10 En même temps, l'animal libère « les compétences-socle ». Il s'agit des compétences qui constituent le socle du développement affectif, émotionnel, social et cognitif. En clair, il y a 5 compétences-socle :

- l'attention visuelle soutenue,

- l'élan à l'interaction

- les comportements affiliatifs 
- l'organisation structurée du geste

- l'imitation

\section{Première compétence-socle : l'attention visuelle soutenue}

Alors, qu'entendre par là ? par là, on entend une attention visuelle focalisée, qui n'est pas interrompue par les événements extérieurs, une attention qui ne « mollit pas », si on peut dire. L'attention visuelle soutenue, les yeux dans les yeux, c'est le contraire de la rêverie où la personne s'abstrait. Au cours de cette interaction les yeux dans les yeux, l'enfant s'ouvre le livre des émotions et des affects de son partenaire. Et l'enfant livre ses propres émotions, et ses propres affects. C'est ce qui lui permet de développer des interactions dites " accordées ", c'est-à-dire dans lesquelles il y a justement, non pas seulement des comportements mais aussi des émotions, des affects et des rythmes. Et ceci est indispensable à l'installation d'un attachement sécurisé (secure). Quand un enfant n'a pas d'attention visuelle soutenue, il se met en position d'éviter le regard de l'autre, et alors ça, c'est la voie royale pour les troubles et les dysfonctionnements à tous points de vue.

12 Alors précisément, dans la relation avec l'animal - les animaux familiers, j'entends - il y en a cinq groupes qui peuvent être qualifiés d'animaux familiers, c'est-à-dire le chien, le chat, le cheval, le dauphin, et le perroquet - quand l'enfant est en interaction avec l'un de ces animaux, il peut rester les yeux dans les yeux avec l'animal, sans que l'animal luimême détourne le regard. Autrement dit. l'enfant peut faire l'hypothèse, peut former l'idée, peut avoir le sentiment, que l'autre l'écoute, que l'autre l'entend, et il peut en même temps formuler des hypothèses ou des certitudes sur ce que l'animal ressent. « oh, toi, je vois bien que tu es comme ceci, je vois bien que tu es comme cela »; et c'est les yeux dans les yeux que ça se fait, ça. Donc, c'est vraiment l'enfant qui s'ouvre le livre des émotions qu'il perçoit ou qu'il croit percevoir, à travers le regard de l'animal.

Hélène Dufau. : Ça serait un apprentissage Je I empathie?

Hubert Montagner: Absolument, on peut appeler ça comme ça, ça peut être un jeu d'empathie, mais l'important c'est que l'enfant le vive comme ça, le croie. Or, ceci est indispensable au développement de la communication multi-canaux, parce que nous, le humains, c'est à travers «les-yeux-dans-les-yeux» que nous développons la communication multi-canaux, c'est à dire que nous agrégeons, nous combinons, aux informations visuelles que nous recevons par le canal visuel, les informations auditives (y compris évidemment celles qui viennent du langage, donc qui sont véhiculées par le langage), les informations du toucher (les informations somesthésiques). les informations olfactives, et toute autre information. C'est finalement œil à œil, c'est le ciment qui agrège les différentes informations, qui fonde ce qu'on appelle la communication multicanaux. Il n'y a pas de communication multi-canaux si une la relation « les-yeux-dans-lesyeux » ne se développe pas. Hélène Dufau. : D’où le fait de ne jamais porter des lunettes noires si on veut communiquer. !.. Hubert Montagner : Ça c'est clair, quelqu'un qui porte des lunettes noires n'est pas un être de communication, c'est évident. Et c'est pour ça aussi que les aveugles ont beaucoup de difficultés à communiquer, beaucoup plus que les sourds, même quand ils maîtrisent bien le langage.

Hélène Dufau. : De leur propre fait, ou du fait de tout le monde? 

établit des relations proximales. Ça, c'est le fondement des relations proximales, qui sont importantes à tous les points de vue, évidemment. C'est grâce à ces interactions proximales que les interactions peuvent être accordées, c'est-à-dire qu'il peut y avoir ajustement des états émotionnels, affectifs et rythmiques. Et ça, c'est indispensable pour qu'un attachement « secure » s'installe et se développe.

Hubert Montagner : du fait de tout le monde, parce que tout le monde s'attend à ce que la communication s'articule autour de «les-yeux-dans-les-yeux ». Et il n'y a rien de plus malaisé dans une situation relationnelle que l'évitement du regard. Et d'ailleurs, les enfant qui évoluent sur une pente pathologique sont des enfants qui sont en déficit d'attention visuelle soutenue « les-yeux-dans-les-yeux ».

Hélène Dufau. : C'est donc un symptôme

Hubert Montagner : Oui, les enfants autistes, les enfants psychotiques, évitent le regard. L'attention visuelle soutenue, c'est aussi l'attention visuelle conjointe qui peut se développer : c'est à dire que si le partenaire de l'enfant réoriente son regard, et a un geste de désignation, une parole de désignation, l'enfant va faire glisser son regard dans cette direction; et réciproquement. Et l'attention visuelle conjointe, c'est aussi une façon de se dire qu'on est sur la même longueur d'onde.

\section{Deuxième compétence-socle, l'élan à l'interaction}

Et pour l'enfant qui est en difficulté avec les être humains - avec sa mère, avec son père, avec sa famille - c'est important qu'il ait affaire à l'animal qui a de l'élan à l'interaction.

Hélène Dufau: Oui, parce que s'il est lui-même replié, ça ne va pas s'arranger pour lui.

Hubert Montagner : Voilà. Et l'élan à l'interaction, c'est ce qui lui permet de mobiliser luimême son propre élan à l'interaction. Chez les chiens, il y a une quête affective permanente, il y a un élan permanent. Les chats, c'est la même chose. Mais les chats sont des alternatifs, ils ont des temps égoïstes et des temps de dépendance obligée et complète vis-à-vis de l'être humain. Et dans leurs temps de dépendance obligée, ils ont tellement d'élan à l'interaction, qu'ils sont invasifs. qu'ils sont intrusifs. qu'ils sont embêtants, on ne les supporte plus. Mais ils ont un élan à l'interaction extrêmement fort. Et lorsque les enfants ont une mère dépressive, une mère angoissée, qui a des troubles de la personnalité, eh bien c'est important qu'ils aient ce partenaire d'élan à l'interaction.

Hélène Dufau: Mais dans ce cas, pourquoi les chevaux, alors? Parce que les chevaux $n$ 'ont pas autant d'élan a l'interaction...

Hubert Montagner : avec les chevaux c'est un peu différent. Ils sont plus grands, ils ont une latéralisation des yeux, ils ont une masse corporelle impressionnante, ils ont une tête impressionnante, ce qui crée de la distance, de la distance sensorielle et interactive. Mais quand ils arrivent à s'apprivoiser mutuellement, là le cheval peut avoir des élans à l'interaction tellement forts que si un enfant siffle le cheval va arriver de loin.

Hélène Dufau : Ça n arrive pas si fréquemment que ça, si ?

Hubert Montagner : En milieu rural, ce n'est pas si rare que ça. Car les chevaux ils sont très attentifs à leur environnement, quand ils perçoivent leur maître soit auditivement, soit visuellement, soit olfactivement, ils arrivent tout de suite. Et ils ont des 
comportements de salutation qui favorisent la proximité corporelle. Donc ils ont aussi un élan à l'interaction ; les chevaux familiers, encore une fois.

\section{La troisième compétence-socle, c'est l'imitation}

Donc tous les animaux familiers imitent ou donnent l'impression qu'ils imitent c'est-àdire que non seulement ils reproduisent des comportements humains, mais qu'ils lui donnent le même sens et la même signification. C'est le sentiment qu'on a, mais ce qui compte, ce n'est pas tellement la réalité, c'est le sentiment que l'homme construit par rapport à ça.

\section{Et puis la quatrième compétence-socle : l'organisation structurée et ciblée du geste}

Évidemment, ce sont des animaux qui n'ont pas de bras, pas de mains, donc qui n'ont pas de gestes. Mais ils ont une organisation structurée au plan corporel. Ce sont sur ce plan des animaux très performants, que ce soit le chien, le chat, le cheval, le dauphin et le perroquet. J'en arrive au dauphin. Le dauphin a une attention visuelle soutenue, y compris quand il est dans l'eau, et quand le partenaire humain est au-dessus. Et quand le dauphin est en milieu aquatique avec un humain, vraiment, il est tout le temps en train de le regarder, et ça c'est d'autant plus fort pour un enfant qu'il a des comportements affiliatifs (dont je parlerai un peu plus loin), c'est à dire qu'il a l'air de rire. Surtout le tursiops.

31 Le dauphin a un fort élan à l'interaction, dès qu'il discrimine la personne familière, il se précipite, et dans le milieu aquatique, ça peut même être gênant quand il vient se frotter...

Le perroquet, qui a aussi des capacités d'attention visuelle soutenue, ou du moins il donne l'impression d'avoir une capacité d'attention visuelle soutenue, parce que avec sa capacité « langagière », il fait s'approcher les personnes, et à ce moment là, il a un regard à la fois mobile et immobile, qui fascine les enfants parce qu'il regarde sans regarder, mais tout en regardant, et tout en parlant. Bon, le perroquet ne comprend sans doute pas le sens, la signification de ce qu'il reproduit, même si c'est dans le même contexte que celui où il l'a entendu mais il a une capacité de mémorisation extraordinaire.

II y a donc l'attention visuelle soutenue, l'élan à l'interaction, l'imitation, l'organisation structurée du geste.

\section{Enfin, les comportements affiliatifs, la cinquième compétence-socle}

je les ai laissés pour la bonne bouche. Ce sont des comportements qui paraissent traduire l'adhésion du partenaire à ce qu'on fait et à ce qu'on dit.

Hélène Dufau : Qui laissent penser que finalement, on est bien d'accord...

Hubert Montagner : C'est tout à fait ça. Donc c'est le sourire, le rire, c'est l'offrande, c'est la sollicitation, ce sont les gestes de désignation, c'est la coopération, ce sont les 
comportements sociaux, quoi... Chez le chien, c'est le chien qui s'est approché, qui va entr'ouvrir la gueule, avec la tête et les oreilles droites, qui va remuer la queue d'une certaine façon, qui va avoir des comportements juvéniles ou infantiles... Donc ça. ça paraît traduire l'adhésion. L'enfant peut lui dire ceci, peut lui dire cela, l'animal a l'air d'accord... Quant au chat, c'est le plus formidable animal qui soit en ce qui concerne le tableau des comportements affiliatifs, parce que il ronronne, aucun autre animal ne ronronne, il se frotte sans arrêt, toutes les parties du corps, il recherche les contacts corporels massifs, quand il set en situation corporelle, sur les genoux ou à plat ventre sur la poitrine, y a son nez qui coule (les écoulements nasaux), qui coule d'émotion, c'est un séducteur complet, le chat.

Le cheval, lui, a ses hochements de tête, il a ses mouvements de pattes, il a ses frottements, il a ses hennissements amicaux, un peu particuliers, il a tout un registre aussi de comportements affiliatifs.

Le dauphin, lui, paraît avoir un rire, il paraît rigoler, comme on dit, il vient se frotter, il vient solliciter, il vient offrir, il offre, tout comme le chat et le chien, et le cheval. Il offre une gamme de comportements affiliatifs aussi. Et le perroquet, lui aussi, a ses comportements affiliatifs. c'est qu'il rit, il ricane, comme il a entendu faire ; c'est perçu comme des comportements affiliatifs.

Voilà donc les cinq groupes d'animaux qui ont développé les cinq compétences-socles qui jouent un rôle important dans le développement émotionnel, affectif, relationnel, social et cognitif de l'enfant. Donc ce n'est sans doute pas par hasard si des relations particulières se sont établies entre ces animaux et les êtres humains, et particulièrement les enfants.

41 Hélène Dufau: Oui. Cyrulnik évoque également cette dimension. Et alors, avec l'adulte, qui est finalement un enfant évolué, vieilli ?...

42 Hubert Montagner: Avec l'adulte ce n'est pas différent, mais souvent, les adultes qui n'ont pas fait la démarche de s'attacher à un animal particulier vont ignorer les animaux, ou vont même développer des conduites agonistiques liées aux animaux « qu'est-ce qu'il fait là, il nous emmerde, il n'a rien à faire là »... donc ils ont des a priori très forts par rapport à ça. Et puis en outre, quand on veut essayer de mêler l'animal à l'homme quant à leurs points de convergence, à leurs analogies, alors là, ça ne passe jamais, bien évidemment... Et puis il y a ceux qui sont toqués, au contraire, de la présence animale à côté d'eux, d'animal de compagnie, ou quelques fois d'animal familier, qui ont eux, tout à fait une autre optique, puisque considérant l'animal, ces animaux que j'ai évoqués, comme des quasi-humains. Donc, ça dépend de ce que décident les adultes

Hélène.Dufau: Alors pour ce qui est de l'idée que. dans les entreprises - puisque notre thème c'est quand même l'organisation - on pourrait utiliser les animaux pour essayer de diagnostiquer, des comportements de personnalité, chez l'adulte, qu'est-ce que vous en diriez, sur le plan théorique?

Hubert Montagner : Ah! mais ça, c'est certain. Mais ça dépend aussi si la relation avec le cheval est « à terre » [à pied], si je peux dire ou si c'est dans le cadre du chevauchement. Car le chevauchement est une situation intéressante, parce qu'il y a une sorte de dialogue tonico-postural qui s'instaure entre l'enfant, (si on parle de l'enfant), ou l'adulte, et l'animal. C'est-à-dire que tout se passe comme si à un changement d'allure, à un changement de mouvement de l'animal, l'humain répondait de façon extrêmement ajustée, et réciproquement. Mais si l'homme développe une émotion comme ceci ou comme cela, il va modifier son comportement, et ceci va être perçu par le cheval, qui va 
s'ajuster. Et alors on va penser que le cheval a compris non pas seulement le message comportemental, mais aussi le sens émotionnel qu'il revêtait.

Hélène Dufau. : Mais quand il s'ajuste, malheureusement, il ne s'ajuste pas toujours dans le sens que l'humain souhaiterait... c'est ça qui est un peu difficile...

Hubert Montagner : Ah! ils sont dans deux univers différents. Alors c'est typiquement un cas où il peut $\mathrm{y}$ avoir ajustement des comportements, mais où il peut ne pas $\mathrm{y}$ avoir interaction accordée, c'est-à-dire des ajustements, non pas seulement des comportements, mais aussi des émotions. On peut avoir l'interaction comportementale, mais l'interaction émotionnelle, c'est autre chose. Autant on peut arriver à distinguer la nature de l'émotion de quelqu'un, et à essayer de rendre l'émotion que j'ai évoqué (qu'il le dit. qu'il l'écrit, enfin, qu'il le manifeste d'une façon ou d'une autre, qu'il l'exprime d'une façon ou d'une autre), autant tout ce qui concerne la vie émotionnelle du cheval, et des animaux, est de l'ordre de la spéculation. Même si Darwin a écrit un remarquable livre là-dessus, The Expression of Emotions in Humans and Animais. Mais ça, c'est de la spéculation pure. On sait pas ce qu'il y a, ce que pense le cheval, on sait pas ce qu'il ressent. En fait, le cheval, quand il modifie son comportement... on est toujours en droit de supposer, mais à condition de dire qu'on spécule, on a toujours le droit, il ne faut pas être intégriste, évidemment...

Mais quand un jour, je sais pas, un cavalier est triste par exemple, eh bien le cheval le décode d'une façon ou d'une autre, parce que les manifestations comportementales, vocales et langagières ne sont pas les mêmes, donc le cheval va distinguer les différences dans le registre d'expression, cette fois-ci. D'expression, c'est-à-dire qu'il lit les comportements, les émotions et les affects. enfin, le monde intérieur. Même chose si l'humain arrive en colère, ou s'il a peur, ou s'il est surpris, ou s'il a du dégoût, enfin, les 6 grandes émotions, ça le cheval les perçoit très bien. C'est un excellent détecteur ou percepteur des émotions humaines, le cheval.

Maintenant, si on rapproche cela de la communication homme/animal, moi, je ne vois pas pourquoi un animal ne pourrait pas avoir un état affectif comme ceci ou comme cela. Le problème, c'est qu'on ne sait pas ce que ça veut dire. On ne sait pas la nature de ce qui paraît être une expression émotionnelle. Qu'est-ce que ça veut dire, qu'est-ce que ça revêt, ça peut être un effet de souffrance qui a l'air d'être un état de tristesse, par exemple, ou de peur, et tout simplement, l'animal réagit à la souffrance. Et il a l'air d'être triste, et il a l'air d'avoir peur, il a l'air comme ceci ou comme cela. Donc, on ne sait pas - on ne sait pas bien qualifier les états intérieurs des animaux. Par état intérieur, j'entends les états psychiques. Donc, c'est ça qui crée la difficulté, en fait. C'est vraiment ça.

C'est comme dans les recherches sur les interactions précoces entre le bébé et sa mère, le bébé et ses différents partenaires, alors on a - c'est Stem, le psychanalyste qui a créé le concept d'alimentent, c'est-à-dire interaction accordée, c'est-à-dire ajustement des émotions et des affects et pas seulement des comportements - pour distinguer justement l'accordage émotionnel et affectif entre le bébé et sa mère. On utilise tous cette expression. Mais dans l'état actuel des choses, on ne sait pas du tout comment explorer la vie émotionnelle et affective d'un bébé. Ça, c'est évident. Donc, c'est du même ordre chez l'animal, on ne sait pas comment explorer ça. Les animaux ne parlent pas, n'ont pas le langage articulé, ils ne dessinent pas, ils ne peignent pas. ils n'ont pas de façon d'exprimer ce qu'ils ressentent. Donc, ça, c'est une source de confusion extraordinaire, et c'est là l'une des pierres d'achoppement de la comparaison entre l'homme et l'animal. Étant bien entendu que chez le bébé de l'homme, il y a le même type de difficulté. 
Hélène Dufau: Alors l'idée que, si on veut détecter par exemple le leadership, au niveau d'un humain, on peut le faire par rapport aux chevaux, parce qu 'il y aura une réactivité adéquate...

Hubert Montagner: On a décrit ça il y a 25 ans, je crois. Quand j'étais à Besançon, professeur à la faculté à Besançon, on a décrit le phénomène du leadership chez les jeunes enfants. Par leader, nous entendons l'enfant qui objectivement a une forte probabilité d'être suivi, et d'être imité par les autres. C'est ça que nous entendons. Ça vient de l'anglais to lead, conduire. Et nous définissons le leader comme cela, et non pas par le degré de probabilité que l'individu a de s'imposer dans les situations de conflit ou de compétition.

\section{Hélène Dufau: C 'est plutôt une attractivité naturelle?}

Hubert Montagner : Voilà, c'est une attractivité objective, si vous voulez. Si on quantifie les choses, il est clair que ce sont toujours les mêmes enfants qui apparaissent en situation de leaders, et il peut y avoir plusieurs leaders dans un groupe. Ils ne s'excluent pas forcément, mais ils ne s'imposent pas par la menace ou l'agression, ou le rejet. Les leaders s'imposent pas les conduites de séduction, les comportements affiliatifs. que j'évoquais tout à l'heure.

54 Hélène Dufau: Ça veut donc dire que si on mettait des adultes en contact avec des chevaux pour détecter leur capacité de leadership, est-ce que vous pensez que ça facilite les choses, que c 'est significatif?

Hubert Montagner : Alors là. on transpose le concept au niveau interspécifique, et donc pour cela, il faut prendre des risques, mais après tout, pourquoi pas? Le problème c'est que l'homme est toujours leader, dans la relation avec un animal, il est toujours leader.

Hélène Dufau : On peut créer les conditions pour que ça ne soit pas le cas...

Hubert Montagner : C'est vrai pour la phase initiale de l'interaction: il est forcément leader dans la phase initiale de l'interaction. Mais ensuite, ça peut être différent, c'est-àdire qu'il peut se trouver que ce soit l'animal qui est suivi, qui est imité par l'humain, c'est évident. Ça. chez l'enfant, c'est absolument caricatural. (Chez les adultes, c'est moins évident). Mais ne sais pas. moi, si un chien familier commence à faire ceci ou cela, eh bien il y a une forte probabilité que l'enfant essaie de faire ceci ou cela. Il n'y a que le chat pour qui ce serait différent : parce que le chat, lui, il a des moments égoïstes tellement forts, qu'on se contente de cette invasion émotionnelle et affective qu'il manifeste lorsqu'il vient au corps à corps. Et personne ne va suivre un chat. D'ailleurs, si on essaie de suive un chat, il va s'écarter, il va éviter, donc chez le chat, c'est impossible. Chez le cheval, oui, ça. peut-être... on connaît des cas comme ça. où c'était le cheval qui prenait les devants, si on peut dire, et l'humain ou les humains qui suivaient.

Hélène Dufau : Mais quand on monte à cheval, on s'aperçoit que souvent, ce n'est pas l'homme qui commande, il se fait manouvrer complètement...

9 Hubert Montagner: Au plan scientifique, ça serait intéressant d'étudier les processus mentaux des humains selon qu'ils acceptent ou qu'ils n'acceptent pas un leadership occasionnel, ou fréquent, ou permanent du cheval. Le cheval qui prend, lui, les initiatives. Alors ça, je crois qu'il y a toute une voie de recherche dans ce domaine là.

Hélène Dufau : Pour vous, peut-on dire qu étudier I interaction enfants/animaux permet de mieux comprendre la condition humaine?

Hubert Montagner: Oui, parce que je vous ai parlé tout à l'heure de communication multi-canaux ; précisément quand la communication n'est pas brouillée par le langage, si 
j'ose dire, l'enfant qui est en interaction avec un chien va donc avoir comme ciment l'interaction les yeux dans les yeux, et c'est là-dessus, autour de ça qu'il va agréger, il va combiner, il va associer les autres informations qu'il reçoit. Donc c'est comme M. Jourdain, il va finir par faire de la prose sans le savoir, c'est-à-dire de la communication multi-canaux sans le savoir. Il va donc construire des processus de communication dans lesquels se trouvent combinés, associés, agrégés, les différents types d'information qui sont reçus.

Hélène Dufau: Et donc ensuite, il peut le transposer plus facilement aux humains, il va outrepasser finalement, sa barrière...

Hubert Montagner : Consciemment ou inconsciemment, il va pouvoir passer à d'autres registres, par la suite. Il va se structurer, finalement, dans un autre monde, et en interaction avec les humains, et alors qu'il pouvait être hyperactif, par exemple, eh bien il peut très bien prendre le temps d'écouter, d'entendre, de voir, de sentir, avant de répondre.

Hélène Dufau : Est-ce que vous pensez justement que ces connaissances là sont vraiment exploitées suffisamment dans les milieux thérapeutiques?

Hubert Montagner: Pas du tout. D'ailleurs, le livre L'enfant et l'animal que j'ai sorti est perçu par les psychiatres aussi bien comme un livre d'éthologie que de pédopsychiatrie. Disons que ça leur a fait découvrir que la relation avec l'animal pouvait structurer, stimuler, un certain nombre de leviers qui ne peuvent pas se structurer dans le cadre de la relation humaine. C'est le cas de l'enfant autiste, de l'enfant psychotique, c'est le cas de l'enfant autocentré, introverti, timide, « caractériel »... Et ils prennent conscience qu'il y a des processus qui sont activés, et structurés, dans le cadre de la relation avec l'animal. Alors qu'ils n'imaginaient pas ça tellement; pour eux, psychiatres, le psychisme humain est unique, et ne peut pas, peu ou prou, trouver des racines dans la psychologie animale. Et ça les trouble, parce qu'ils n'avaient pas pensé à ça.

Hélène Dufau: Autre question, celle de l'institution. Est-ce que vous pensez qu un jour on va utiliser les animaux dans le cadre des institutions de manière systématique, massive?

Hubert Montagner : C'est déjà fait. Il y a un livre qui vient de sortir à ce sujet. Le chien, partenaire de vies, sous la direction du Dr Didier Vernet (secrétaire général de l'association dont j'ai été le président pendant longtemps. l'AFIRAC. Association française d'information et de recherche sur l'animal de compagnie), à la préface duquel j'ai contribué.

Hélène Dufau: Qu 'est-ce que vous diriez des SAC (nouveaux animaux de compagnie, voyez: serpents et autres mygales). Est-ce qu 'on ne peut pas commettre dans ce domaine des erreurs grossières du point de vue de ce que vous énoncez, justement

Hubert Montagner : Ces animaux n'ont pas les compétences socles que j'ai évoquées. Pas même les singes. Le problème des singes, c'est l'évitement du regard. Il ne faut pas le regarder, c'est un fondement de la vie sociale chez les singes. Ce qui veut dire qu'il ne peut pas y avoir le sentiment que l'animal partage ses émotions. Il ne peut pas y avoir d'interaction accordée avec un singe, et je me souviens de ce qu'ont dit et qu'ont écrit deux amis qui étaient les Gardner, qui ont appris le langage des sourds-muets américains à des chimpanzés (Washoe). Il a fallu s'en débarrasser, parce qu'elle devenait agressive, elle ne pouvait plus développer des comportements affiliatifs à partir de la préadolescence (à cause du regard). Et donc, ils ont un élan à l'interaction quand ils sont petits, mais arrivés à la puberté, au contraire, ils évitent la rencontre ils la fuient. Les 
comportements affiliatifs. certains ont décrit les sourires chez les singes, et notamment chez les chimpanzés... On peut dire tout ce qu'on veut dans ce domaine là. hein, c'est comme un bébé, un bébé peut faire une grimace comme ceci, sa mère va dire « oh! il a souri », heu, bon!! Les mimiques faciales des chimpanzés, quelquefois, peuvent donner l'impression de sourire... Mais...

Hélène Dufau : Autre exemple, les hamsters...

Hubert Montagner : Oui. ils jouent un rôle important dans certains aspects : ils ont un élan à l'interaction, ils ont des comportements affiliatifs. mais ils n'ont pas «les yeux dans les yeux ». ils n'ont pas la capacité d'imiter, c'est très différent. Bon, les relations corporelles avec un lapin par exemple, ça permet d'instaurer la sécurité affective, donc le premier étage de la fusée. Deuxième étage de la fusée, ça permet de libérer les émotions, mais en revanche, ça ne permet pas de mettre en place des compétences sociales...

Hélène Dufau : Donc, ça sera incomplet...

Hubert Montagner: Oui car il faut parler du troisième étage, qui est quand même important, c'est-à-dire le satellite qui est lancé dans l'espace, c'est-à-dire la structure dite intelligente. Quand il y a libération des émotions et des compétences-socles, à ce moment-là, le troisième étage peut être libéré, c'est-à-dire les processus cognitifs, les compétences intellectuelles, et l'imaginaire, en interaction les uns avec les autres. Et ça, ça donne la créativité. Le cerveau est ainsi fait.

74 Si l'enfant vit dans l'insécurité, dans la peur, il ne peut pas libérer ses compétences-socle, il faut le libérer, déminer le terrain. Par rapport aux adultes, on peut obtenir le même genre de déblocage, mais c'est plus difficile, parce qu'ils sont construit déjà comme ça. L'enfant est en cours de construction. Les structures nerveuses centrales sont plus plastiques, et les comportements plus flexibles.

Hélène Dufau : Mettre des animaux dans des structures psychiatriques, ce serait tout bénéfice?

Hubert Montagner : Absolument oui. Rien que le fait de placer un individu sur le versant de la sécurité affective, c'est important. Le fait qu'il n'ait plus peur de son environnement et de lui-même, rien que ça. Et ça lui permet de libérer ses émotions, de libérer les compétences-socles, donc. là. il devient un être humain acteur.

Hélène Dufau: C 'est moins ennuyeux que les neuroleptiques, mais moins lucratif pour l'industrie pharmaceutique...

Hubert Montagner : Je pense que ça peut remplacer les neuroleptiques... J'ai vu des cas d'enfants dits "hyperactifs " par exemple, dans une classe où il y avait un chat ou un chien, eh bien, certains enfants n'apparaissaient pas du tout hyperactifs...

Hélène Dufau : Est-ce qu 'on peut comprendre la spécificité de l'homme à travers tout ça ? qu 'est-ce que ça aide à détecter par rapport aux animaux? Hubert Montagner: La spécificité de l'homme, à mon avis, c'est sa capacité à donner plusieurs sens et plusieurs significations à un message donné. Selon son vécu, selon les expériences individuelles, selon la situation, selon le contexte donc, ça ne gêne pas un humain de penser des choses contradictoires en même temps.

Hélène Dufau :Ça le gêne, quand même. Il y arrive, mais peut-être que parfois, ça le perturbe...

Hubert Montagner : Je veux dire que c'est possible. Il en est capable. Un humain peut penser plusieurs choses différentes d'un message donné. Ça, c'est l'une des premières spécificités de l'homme, ce qui n'est pas le cas chez l'animal. Ce qu'est capable de faire un 
animal plus complexe, c'est de mentir. Un chien est capable de mentir ; et un cheval aussi. Donc, il est capable finalement de conduire un humain à avoir deux idées différentes, mais successivement, dans le temps, mais pas en même temps. Alors qu'un humain peut très bien penser deux ou trois choses différentes à propos d'un signal ou d'un message donné. bon... pour faire avancer le bateau... Et puis il y a les neurophysiologistes purs et durs, qui sont très frustrés à l'heure actuelle, parce qu'il n'y a aucun neurobiologiste qui est capable d'expliquer comment fonctionne le cerveau. Alors ce que je vous ai dit tout à l'heure à propos des trois étages de la fusée, effectivement, ça les laisse interloqués. Ils ne peuvent pas dire non, c'est comme ça que ça fonctionne; jusqu'à preuve du contraire. Mais là où ils sont en difficulté, c'est qu'une chose est d'arriver à doser des molécules dans telle ou telle structure du système nerveux central, et autre chose est de comprendre le fonctionnement du cerveau. Ils s'attachent à la technique, ils restent dans le domaine technologique. Telle ou telle image du cerveau qui s'allume comme ceci, comme cela., comment interpréter cela? qu'est-ce que ça veut dire? est-ce qu'il y a tellement de passage qu'elle s'allume, ou alors c'est une structure au stade de l'intégration, et donc elle travaille plus que les autres? Et ça, ça ne veut rien dire. Les neurosciences sont en grave difficulté car on ne sait pas vraiment dire comment fonctionne le cerveau.

Hélène Dufau: Une approche comme la mesure fine des codages biochimiques, c'est extrêmement réducteur, comme démarche.

Hubert Montagner : C'est très très réducteur, et le cerveau fonctionne de façon globale. Quand je fais ça [geste du bras], c'est tout mon cerveau qui fonctionne, et ça n'a plus de sens de faire comme on faisait il y a trente ans. On enseignait qu'il y avait telle structure qui avait telle fonction, etc.. la zone de Broca, si, elle joue un rôle, si, mais elle n'est pas la seule. On sait bien que la neuropsychologie a ses limites, car on a pu montrer... les américains ont sorti toute une série de papiers il y a 45 ans maintenant, sur des splitbrains, où il apparaissait qu'il y avait une redistribution des cartes...

90 Et je leur dis : savoir qu'il y a là des neurones qui sécrètent de la dopamine,... tel médiateur neurochimique, au plan scientifique pur et dur, c'est important de savoir que ça se trouve dans telle structure plutôt que dans telle autre, et d'essayer de voir à quoi ça 
peut servir au plan de la santé de telle plage neuronale, de telle autre plage neuronale, bien sûr que ça m'intéresse, mais en ce qui concerne l'expression comportementale en relation avec la fonction cérébrale, qu'est-ce que ça apprend ? Vous aurez beau injecter de la dopamine à un individu, vous n'allez pas faire ceci, vous n'allez pas faire cela... Enfin, dans l'état actuel des choses, la neurobiologie ne peut pas expliquer le fonctionnement du cerveau. Le seul qui donne une explication qui tienne un peu la route, c'est Damasio, qui lui, justement, a montré à quel point on ne peut pas comprendre le fonctionnement cérébral sans prendre en compte les émotions.

91 Hélène Dufau: Pour la démarche thérapeutique, en fait, soigner les gens avec des thérapies qui impliquent le corps, qui a enregistré les émotions, $c$ 'est sûrement plus efficace que d'être dans le langage pur?

Hubert Montagner: Oui. effectivement, qui implique l'interaction et la globalité de la personne, l'interaction, c'est le cerveau réconcilié avec le corps... et le cerveau réconcilié avec le corps. C'est-à-dire en intégrant, en fait, la libération des émotions, et la libération des compétences-socles, ça va en même temps et c'est interactif. 